\title{
Am Ende der modernen Sicherheit: William James über religiöse Erfahrung
}

\author{
Markus Riedenauer \\ Lehrstuhl für Philosophische Grundfragen der Theologie \\ Katholische Universität Eichstätt-Ingolstadt \\ Pater-Philipp-Jeningen Platz 6, 85072 Eichstätt, Deutschland \\ markus.riedenauer@ku.de;www.ku.de/thf/philosophie/
}

\begin{abstract}
At the End of Modern Security: William James on Religious Experience

William James defends religious belief as a reasonable option against a kind of widespread agnosticism which he calls scientific absolutism, and against the dogmatism which he sees in the natural theology of his time. On the basis of his collection of essays "The Will to Believe", the article reconstructs his arguments and the epistemological foundation of his famous treatment of religious experience in "The Varieties of Religious Experience". James' pragmatistic approach, which he calls radical empiricism, resists the exclusion of "mystical" experiences of conversion and redemption, and of religious faith from the realm of reasonable attitudes. Experiences of the astonishing gift of being, of trust and openness, courage and motivation to endure life's evils can validate religious faith.

In so far as modern rationality with its highest expression in the sciences is rooted in an existential quest for security, the underlying attitude towards life unnecessarily prevents personal experiences of the divine and salvation and unreasonably devaluates attitudes of faith. James defends the desiring nature of human beings and opens up the space for legitimate religious experience.
\end{abstract}

\section{Keywords}

religious experience - belief - radical empiricism - scientific agnosticism - pragmatistic epistemology - natural theology - dogmatism - motivation - divine presence 


\section{Neuansatz der Religionsphilosophie gegen verschiedene Dogmatismen}

William James (1842-1910) gilt als einer der Begründer einer Religionstheorie, welche religiöse Erfahrung ernst nimmt und ihr eine fundierende Rolle zumisst - und zwar für Religion überhaupt. Es gebe „bei allen Diskrepanzen unter den Glaubensbekenntnissen einen gemeinsamen Kern, von dem sie einmütig Zeugnis ablegen“, nämlich ein Unbehagen und eine Befreiung oder Heilung davon - „das Wesen ihrer religiösen Erfahrung.." ${ }^{1}$ Dieser Ansatz ist bis heute fruchtbar. Friedo Ricken etwa baut seine Religionsphilosophie auf der Kritik Wittgensteins an der Krankheit des Verstandes auf, welcher nach dem Muster der naturwissenschaftlichen Methode immer nach dem Wesentlichen, nach Allgemeinheit strebt, ${ }^{2}$ wogegen James den "religiösen Grundakt" in subjektiver Erfahrung fundierte.

Das religionsphilosophische Heilmittel gegen diese Krankheit findet sich bei William James, es trägt das Etikett ,Die Vielfalt der religiösen Erfahrung‘. Wie Wittgenstein die vielfältigen Sprachspiele beschreibt, so muß die Religionsphilosophie die vielfältigen Formen des religiösen Bewußtseins und seiner Äußerungen beschreiben. ${ }^{3}$

Das leistete James in The Varieties of Religious Experience. Gerd Haeffner sieht die Herkunft schon der Wortprägung „bei William James, der sie in den Titel seines Werks ,The Varieties of religious experience (1901-02) gesetzt hat [...]. Denn seither hat sich dieser Begriff mehr und mehr in verschiedenen Sprachen verbreitet, während er vorher nicht (oder kaum) bezeugt ist.." ${ }^{4}$ Die Neuerung ergibt nur dann einen Sinn, wenn experience / Erfahrung nicht auf Sinneswahrnehmung beschränkt wird, was für unseren deutschen Sprachgebrauch ohnehin nicht gilt. Gegen Ende des 19. Jahrhunderts freilich war die Situation noch vom sensualistischen Empirismus und Positivismus (unter Einflüssen von Hume, Comte und Condillac) geprägt - ein Denken, welches nach

1 The Varieties of Religious Experience tragen den Untertitel „A Study in Human Nature“, London - New York - Toronto 1929; zitiert als " $V R E^{\prime}{ }^{.}$, „WtB“ = The Will to Believe and other essays in popular philosophy, New York etc. 1927. „Vielfalt“ = Die Vielfalt religiöser Erfahrung. Eine Studie über die menschliche Natur, Berlin 2014. Das Zitat findet sich in VRE p. 490 / Vielfalt p. 487; vgl. auch VRE p. 486 / Vielfalt p. 484.

2 Wittgenstein 1984, pp. 37-39; siehe Ricken 2003, p. 17.

3 Ricken 2003, p. 17.

4 Haeffner 2004, pp. 32 et seq. 
Henri Bergson, ${ }^{5}$ dem James viel verdankt, an der Oberfläche verblieb, wogegen auf die im Bewusstsein erfahrene tiefere Wirklichkeit zurück zu gehen sei. Die geistesgeschichtliche Situation in Nordamerika im 19. Jahrhundert war von den empirischen Wissenschaften geprägt, im Vergleich womit die rationalistischen Theologien, die James „scholastischen Absolutismus“ nennt, nur ein Nischendasein fristeten. In der Philosophie dominierte außerdem der Idealismus in der Hegelschen Variante einerseits, ${ }^{6}$ in Form des Transzendentalismus andererseits, seit 1836 in Boston der „Transcendentalist Club“ gegründet wurde, um die Würde des Menschen mitsamt Phantasie, Gefühl und Wille zu verteidigen: In wirkmächtiger Weise propagierte R.W. Emerson die „ÜberSeele" des einen universellen Geistes.

Diese Stichworte müssen genügen, um den allgemeinen Kontext von James' Neuansatz zu vergegenwärtigen. „Wie kaum ein anderer hat James die Spannung zwischen Religion und naturwissenschaftlichem Denken erfahren." Er hatte Physiologie, Chemie und Medizin studiert, an einer zoologischen Expedition teilgenommen, er lehrte Anatomie und Physiologie und führte die experimentelle Psychologie an der Universität ein. Von der ungebrochen erfolgreichen Naturwissenschaft, die mit der Evolutionstheorie offenbar dabei war, hergebrachte Argumente für einen Schöpfungsglauben erfolgreich zu widerlegen, waren religiöse und für Religion offene Denkansätze durch einen Graben getrennt. Die James bekannte Religionsphilosophie (im weiteren Sinn, welcher philosophische Theologie einschließt) bestand in angelsächsischen idealistischen Systemen und in Neuscholastik - beide erschienen ihm abstrakt, leer und steril. Die Prätention, die Welt von einem Standpunkt oder Prinzip aus in den Griff zu bekommen, lehnte er als „Monismus“ ab. Dagegen richtet sich sein Neuansatz bei "religious experience“, in den Kapiteln XVI und XVII von VRE als "mysticism“ (Mystik) entfaltet, um die Vielfalt, Lebendigkeit und Tiefe der Welt und ihrer Erfahrung zu retten.

Wir sehen demnach bei James eine dreifache Frontstellung, gegen den Idealismus, gegen die scholastisch verhärtete philosophische Theologie und vor allem gegen eine Naturwissenschaft, welche die Grenze zum Naturalismus übersprang im Bewusstsein, die endgültige Rationalität zu verkörpern. ${ }^{8}$

5 Vgl. den Beitrag von Gerrit Steunebrink in diesem Band.

6 „Hegelism [...] may really be reckoned one of the most powerful influences of the time in the higher walks of thought. And there is no doubt that, as a movement of reaction against the traditional British empiricism, the hegelian influence [...] is doing service of a certain kind.“ („On some Hegelisms“, in WtB pp. 263-298, zitiert p. 263).

7 Ricken 2003, p. 57.

8 James kritisiert auch die monistische Philosophie etwa Ernst Haeckels, der einfach das Physische vergöttliche: „Even the laws of physical nature have, in these positivistic times, 
Schon im Vorwort zur Aufsatzsammlung WtB kommt zum Ausdruck, wie James die Sonderstellung seiner Theorie sieht, im Gegensatz zu einem selbstzufriedenen Agnostizismus aus rationalistischen Motiven: Er erwartet, die meisten seiner professionellen Kollegen würden über den Irrationalismus seiner religionsphilosophischen Ansicht lächeln. Und wenn er in den ersten vier Aufsätzen die Legitimität religiösen Glaubens verteidigt, würden manche „rationalizing readers" das als traurigen Missbrauch seiner professionellen Position betrachten $(W t B \times)$. Jene Leute dächten offenbar im Modell einer dem unvernünftigen Glauben zuneigenden Menschheit, denen gegenüber die intellektuelle Elite eine aufklärende Aufgabe habe. Wer indessen Religion verteidige, verrate damit seine professionelle, insbesondere professorale Aufgabe.

James räumt ein, dass es gegenüber einem populären Publikum nötig oder nötiger sein könnte, die Menschen dem „Nordwest-Wind der Wissenschaft" auszusetzen, um ihre Geistesschwäche und Barbarei wegzublasen. „But academic audiences, fed already on sciences, have a very different need. Paralysis of their native capacity for faith and timorous abulia ${ }^{9}$ in the religious field are their special forms of mental weakness" (WtB x).

Keine wissenschaftliche Methode könne bewirken, dass man die rechte Mitte trifft zwischen den Gefahren, zu wenig und zu viel zu glauben - das sei eine Aufgabe, die mit Weisheit zu bewältigen ist. Dann nimmt James einen weiteren möglichen Einwand vorweg, der um 1900 näher gelegen haben dürfte als im 21. Jahrhundert: Das Bemühen, den geistigen Raum für individuellen Glauben zu verteidigen, sei unnötig, denn im Zeitalter der Toleranz werde die Wissenschaft keinen Glauben angreifen - „provided we enjoy it quietly with our friends and do not make a public nuisance of it in the market-place“ (WtB XI). Inzwischen wurde der Marktplatz leider oft zum Ort von mehr als nur „Belästigung“ durch Selbstmordattentate - sei es in Burkina Faso, Bagdad oder Berlin. Die Radikalisierung von religiösem Fanatismus dürfte auch eine Revitalisierung von sich auf Wissenschaft stützendem Atheismus und Religionskritik motivieren. Beidem gegenüber erscheint Religionsphilosophie wieder verstärkt als notwendig. Doch kommen wir zurück zum geistesgeschichtlichen Kontext, aus dem heraus William James zu interpretieren ist.

been held worthy of divine honor and presented as the only fitting object of our reverence." („Reflex action and Theism“, in WtB p. 121).

9 Übersetzbar mit: ängstliche Trägheit, feige Entscheidungsschwäche. 


\section{Religiöse Erfahrung und Bewährung im nichtreduktionistischen, pragmatischen Empirismus}

James etablierte eine Sicht auf Religion, die ganz in Erfahrung gründet, durchaus subjektiver und nicht objektiver Art - aber nicht als Antithese zur Naturwissenschaft, als Mystik im schlechten, nämlich irrationalen Sinn. Vielmehr wollte James eine andere Art von Rationalität und Erforschbarkeit religiöser Erfahrung aufweisen in ihren Folgen für die Lebenspraxis - dem pragmatischen Ansatz entsprechend. Eine Theorie gilt für ihn insoweit als verifiziert, als sie neue Erfahrungen in kohärenter Weise integrieren kann. Dafür musste der Erfahrungsbegriff gegenüber der Tradition von David Hume erweitert werden, sodass auch Relationen erfahrbar sind, und jeder Determinismus abgewehrt wird. ${ }^{10}$ Zwar müssten die Probleme der Philosophie in Begriffen formuliert sein, die alle aus Erfahrung stammen, doch ohne sensualistische Einschränkungen a priori. Unter diesen Voraussetzungen bezeichnete sich James als „radikalen Empiristen" und wir werden sehen, wie das zu verstehen ist.

Schon 1896 (einige Jahre vor der Publikation von VRE und Pragmatism) meinte James, religiöse Hypothesen würden durch das Leben der Gläubigen verifiziert oder falsifiziert - im Prinzip nicht anders als in der Wissenschaft: „The truest scientific hypothesis is that which, as we say, 'works' best; and it can be no otherwise with religious hypotheses" (WtB XII). Nach dem pragmatischen Ansatz wird jede Erkenntnis als eine Hypothese aufgefasst, die sich bewähren muss - für den Einzelnen wie durch die Einzelnen in der Geschichte, welche alle Hypothesen testet. Die moderne Wissenschaft gehört zu diesem Testprogramm, welches Religion in der gegenwärtigen Zeit zu durchlaufen hat.

Die Wissenschaft von der Religion müsse die Kritik der Geschichte an religiösen Annahmen nachzeichnen und zeigen, welche dann den Praxistest bestanden haben. In der zu fördernden Konkurrenz der Religionen würde sich schließlich das „survival of the fittest“ ereignen." Die Wissenschaft habe gar nichts zu fürchten von einer pluralen, lebendigen Religiosität auf deren eigenem Felde, denn die Glaubensweisen, welche die wissenschaftlichen Ergebnisse integrieren, würden den Test letztlich am besten bestehen. Voraussetzung ist freilich, anzuerkennen, dass manche religiösen Hypothesen wahr sein könnten. Damit stellt sich James ausdrücklich gegen die „plenty of scientists who would deny that dogmatically" (WtB XIII), die dann tatsächlich

\footnotetext{
10 Siehe „The Dilemma of Determinism“, in WtB pp. 145-183.

11 "Meanwhile the freest competition of the various faiths with one another, and their openest application to life by their several champions, are the most favorable conditions under which the survival of the fittest can proceed“ (WtB p. XII).
} 
Religion als grundsätzlich nicht wahrheitsfähig aus dem öffentlichen Diskurs verdrängen wollen müssen, was er wissenschaftlichen Absolutismus nennt.

Ein Seitenblick auf die neueste Renaissance der Debatte um „Religion in der Öffentlichkeit" - so einer der Aufsätze von Jürgen Habermas, einem Kristallisationskern der aktuellen Diskussionen über das Verhältnis von Wissenschaft, Politik und Religion (Habermas 2005) - zeigt eine Parallele: James und Habermas meinen, rationalistischer Szientismus könne mit den gefährlichen Herausforderungen durch Religionen ebensowenig gut zurechtkommen wie mit dem positiven Potential, das ernst genommene und in einen vernünftigen öffentlichen Diskurs eingebundene Religion entfalten kann. „Religious fermentation is always a symptom of the intellectual vigor of a society; and it is only when they forget that they are hypotheses and put on rationalistic and authoritative pretensions, that our faiths do harm" (WtB XIII). Die religiösen Ideale und der "Über-Glaube“ seien indessen eigentlich das Interessanteste und Wertvollste an einem Menschen, einer Nation, einer Epoche.

In religionsphilosophischer Hinsicht argumentiert James in $W t B$ gegen einen Agnostizismus als Folge des wissenschaftlichen Absolutismus auf der einen Seite und gegen die rationalistische Metaphysik auf der anderen Seite. Beide sind für ihn Formen von Dogmatismus. James wendet sich, auch in polemischer Weise, gegen rationalistische Metaphysik und betont religiöse Erfahrung, weil er Empirist ohne Reduktionismus sein will. Doch was motiviert seine neue Legitimation religiöser oder "mystischer" Erfahrungen? Der Grundgedanke scheint zu sein, dass die abgelehnten dogmatisch verengten Systeme die Realität verkürzen, ihre Tiefe und Dynamik verdecken. Man könnte auf den ersten Blick den Eindruck haben, dass er für seinen persönlichen $\mathrm{Zu}$ gang zum Göttlichen eine neue Theorie, jenseits der Gottesbeweise und der mittels Deduktion gewussten Eigenschaften Gottes suchte und entwickelte. Jedoch bezeichnete er sich selbst als mystisch unbegabt ${ }^{12}$ - heute würde er vielleicht auch „religiös unmusikalisch“ sagen. Die Frage, wodurch sein erfahrungsbetonter antirationalistischer, „reiner“ und „radikaler“ Empirismus letztlich motiviert ist, welche metaphysische Grundintuition sich so äußert, will meine im Folgenden entwickelte These beantworten: James argumentiert gegen jedes Denken aufgrund von übermäßigem und fehlplatziertem Sicherheitsstreben; er will die Wirklichkeit verstehen ohne die Risiken eines offenen,

12 Z.B. im Brief an Edwin D. Starbuck von 1904: „I have no mystical experience of my own, but just enough of the germ of mysticism in me to recognize the region from which their voice comes when I hear it“ (James 1920, p. 209). Ähnlich schreibt er an James Leuba: „I have no living sense of commerce with a God“ (James 1920, p. 211). 
denkenden Existierens auszuschließen. Das Universum ist für James ein plurales „Multiversum“ von Gegensätzen und Widersprüchen, von Kampf und Widerstand, von politischem und auch prophetischem Durchsetzen und Gestalten, „eine endliche und unvollendete Welt, beständig offen für Neues, durchsetzt von Freiheit."13 Denn nur in einer so verstandenen Wirklichkeit können strebende und handelnde Wesen wirklich zu Hause sein. ${ }^{14}$

\section{Erkenntnistheoretische Grundsätze angesichts der vielfältigen} Wirklichkeit

Diesen Interpretationsansatz verfolgen wir zunächst anhand des Sammelbandes WtB. Dessen Titel "The Will to Believe“ - zugleich der Titel des ersten Essays - ist missverständlich und gegen tatsächliche Missverständnisse (im Sinn eines dezisionistischen Glaubenwollens) stellte James später selbst klar, dass es um das Recht zu glauben geht, ${ }^{15}$ oder besser um die intellektuelle Legitimität eines Willens zum Glauben. Wer genau liest, konnte freilich schon zu Beginn des Essays zur Kenntnis nehmen, er sei „an essay in justification of faith, a defence of our right to adopt a believing attitude in religious matters, in spite of the fact that our merely logical intellect may not have been coerced." ${ }^{\prime 16}$

Im Sinne seines „radikalen Empirismus“ behandelt James in diesem Essay einen Glaubensinhalt als Hypothese. Im Vorwort schreibt er, die hier gesammelten Vorträge drückten ungefähr seine philosophische Einstellung aus. Die Erkenntnistheorie seines „radical empiricism“ betrachtet Schlussfolgerungen über Fakten als Hypothesen, die durch zukünftige Erfahrungen modifizierbar sind. „Radikal“ markiert für James einen Unterschied zu halbherzigen Empirismen wie Positivismus, und Naturalismus, weil er den diesen zugrundeliegenden Monismus nicht als Dogma akzeptieren kann, sondern ihn selbst als eine Hypothese sieht. Die Wirklichkeit weist indessen mehr als nur eine Dimension auf und erfordert, sich erkennend offen zu halten - als Subjekt, d.h. nicht nur mit theoretischer Vernunft, sondern auch mit praktischer

\footnotetext{
13 McCool 1990, p. 769.

14 Das risikobereite Existieren entspricht dem pragmatischen Ansatz, weil nach der Arbeit der theoretischen Vernunft die praktische aufgrund der Strebensnatur ihren Sinn sucht. Ihr erscheint die kognitive Beruhigung des Materialismus und Agnostizismus „irrational to the practical third of our nature [...] we can never volitionally feel at home“ („Reflex Action“, in $W t B$ p. 126).

15 Siehe Ricken 2003, p. $5^{8}$.

$16 \quad W t B$ pp. 1 et seq.
} 
Vernunft und Gefühlen. ${ }^{17}$ Die Realität darf eine metaphysische Dimension haben. ${ }^{18}$ Der wissenschaftliche Zugang zur Welt ist unpersönlich, will nur Objektives und Allgemeines gelten lassen. Das ist für James oberflächlich ("shallow"). „The reason is that, so long as we deal with the cosmic and the general, we deal only with the symbols of reality, but as soon as we deal with private and personal phenomena as such, we deal with realities in the completest sense of the term" (VRE p. 498). Damit wird die Lebenswelt als primäre Welt rehabilitiert; der Unterschied zur Abstraktheit der begriffenen Welt liegt in ihrer vollständigen Wirklichkeit. Religion betrifft "personal destinies“ und hält so Fühlung mit „absolute realities“ (VRE p. 503 / Vielfalt p. 483). Das Selbst ragt in diese größere Dimension hinein. Erlöst werden kann der Mensch durch Identifikation mit dem "höheren“ Teil seiner selbst, von dem mehr im Universum wirksam ist, außerhalb des Menschen - aber mit dem Angebot, sich dorthin zu retten.

Vielleicht wäre der „radikale“ Empirismus besser als „wurzelhafter, ursprünglicher" Empirismus zu bezeichnen. Ausgangspunkt und Boden der subjektiven Erfahrung ist die Vielfalt in der Welt. Hierin liegen die Wurzeln; dem gegenüber ist jede Einheit die Frucht geistiger Arbeit, während eine absolute Einheit (der ganzen Wirklichkeit) ein Grenzbegriff ist. Indessen: Auch nach aller Verstandesarbeit bleiben die endlichen Fakten opak, bleibt vieles unvermittelt und unerklärt.

The negative, the alogical, is never wholly banished. Something - 'call it fate, chance, freedom, spontaneity, the devil, what you will' - is still wrong and other and outside and unincluded, from your point of view, even though you be the greatest of philosophers. Something is always mere fact and givenness. ${ }^{19}$

Auf das fundamentale Gegebensein kommen wir noch unter Kapitel 6 zurück. Es gibt keine komplett integrative geistige Perspektive, darum bleibt das

17 „Damit wendet er sich insbesondere gegen einen Szientismus, der sich selbst zwar als erfahrungsbasiert begreift, dessen Begriff der Realität jedoch ein Konstrukt der Erfahrungswirklichkeit darstellt, das insofern defizitär bleibt, als er von allen subjektiven Elementen der Erfahrung systematisch abstrahiert. Eine möglichst reichhaltige Auffassung der Wirklichkeit im Ganzen muss stets darauf bedacht sein, ihren Ausgang immer wieder bei der konkreten subjektiven Erfahrung zu suchen, da uns hier die Wirklichkeit in ihrer vollständigsten Form gegeben ist“ (Thörner 2011, p. 217).

18 „Dabei geht es James nie darum, Metaphysik aus dem Diskurs zu verbannen: Im Gegensatz zum Positivismus plädiert James für die Wiederbelebung des metaphysischen Denkens durch seine interpretative Anbindung an die Alltagsrealität" (Krämer 2006, p. 45). $W t B$ viri. Vgl. „Reflex Action“, in WtB p. 143. 
menschliche Verstehen auf die Grunderfahrungen angewiesen. Das Universum ist wild und die Natur ganz ein Wunder (WtB VIII). Radikaler Empirismus nimmt Vielfalt an als permanente Form der Welt. „There is no possible point of view from which the world can appear an absolutely single fact."JJames kritisiert hier die positivistische Reduktion der Welt auf Fakten in ihrer eigenen Sprache und die Beispiele im Folgesatz zeigen, wie er die eindimensionale Faktenwelt aufsprengt: „Real possibilities, real indeterminations, real beginnings, reals ends, real evil, real crises, catastrophes, and escapes, a real God, and a real moral life, just as common sense conceives these things." ${ }^{20}$

Im Sinn einer pragmatischen Erkenntnistheorie macht James in WtB zunächst vier Unterscheidungen, auf denen sein Argument gegen die szientistische Bestreitung der Legitimität religiösen Glaubens beruht:

Unterscheidung 1: Eine lebendige Hypothese ist subjektiv eine reale Möglichkeit für das Individuum, "measured by his willingness to act" (WtB p. 3) - im Gegensatz zu einer toten Hypothese.

Unterscheidung 2a: Optionen (zwischen Hypothesen) sind tot oder lebendig - nämlich dann, wenn beide alternativen Hypothesen lebendig sind.

Unterscheidung 2b: Eine Option ist genuin und unausweichlich (bei vollständiger Disjunktion der Hypothesen) oder aber vermeidbar.

Unterscheidung 2c: Sie ist bedeutsam (irreversibel oder einmalig, es geht jedenfalls um Wichtiges) oder aber trivial.

Die Option zu glauben ist nach James nun bedeutsam, genuin und (im Rahmen der eigenen Tradition) an sich lebendig, obwohl sie von szientistischen Vorurteilen erwürgt werden kann.

\section{$4 \quad$ Kritik rationalistischer natürlicher Theologie}

James geht davon aus, dass die moderne Wissenschaft und die Kenntnis des Leidens in der Welt es verunmöglichen, die Natur als direkten Ausdruck eines gütigen göttlichen Schöpfers anzusehen und daraus ein Beweismittel für Gott zu gewinnen. Die natürliche Theologie im naiven Sinn rechtfertige eine spirituelle Weltsicht mit einer ordnenden Kraft in der Natur und entsprechender Theodizee eines moralischen und intelligenten Schöpfers. Sie gehe angesichts naturwissenschaftlichen Wissens und angesichts der Übel der Welt unvermeidlich bankrott.

$20 \quad W t B$ p. Ix. Zum Begriff des Realen und der Berufung auf den common sense bei James siehe die gründliche Untersuchung von Krämer 2006, bes. pp. 81-142. 
But those times are past; and we of the nineteenth century, with our evolutionary theories and our mechanical philosophies, already know nature too impartially and too well to worship unreservedly any God of whose character she can be an adequate expression. Truly, all we know of good and duty proceeds from nature; but none the less so all we know of evil. Visible nature is all plasticity and indifference, - a moral multiverse. ${ }^{21}$

James zählt sich selbst zum Kreis der Menschen, für die die natürliche Religion, also Glauben aufgrund der Naturordnung, definitiv bankrott ist. ${ }^{22}$ Demgegenüber ist sein Beweisziel, zu plausibilisieren "that we have a right to believe the physical order to be only a partial order; that we have a right to supplement it by an unseen spiritual order which we assume on trust, if only thereby life may seem to us better worth living again" (WtB p. $5^{2}$ ). Aus systematischer Sicht müsste es problematisiert werden, wie James hier platonisiert ${ }^{23}$ durch Unterscheidung einer anderen Welt, die in unserer nur oberflächlich und verschleiert erscheine - und sie noch mit dem unglücklichen Ausdruck „supplementary“ versieht. ${ }^{24}$ Aber im Rahmen dieses stärker historisch orientierten Beitrags sei das nur als Indiz dafür genommen, welche Kraft das naturalistisch geschlossene Weltbild am Ende des 19. Jahrhunderts hatte, sodass zunächst nur dessen „Ergänzung“ mit einer anderen Welt möglich erschien. ${ }^{25}$

21 "Is Life Worth Living“, in WtB p. 43. Das Ignorieren des Bösen kann mit der „Religion des gesunden Geistes“ in Vorlesungen IV-V von VRE verglichen werden; „there is no doubt that healthy-mindedness is inadequate as a philosophical doctrine, because the evil facts which it refuses positively to account for are a genuine portion of reality; and they may after all be the best key to life's significance, and possibly the only openers of our eyes to the deepest levels of truth" (VRE p. 162).

22 „For such persons the physical order of nature, taken simply as science knows it, cannot be held to reveal any one harmonious spiritual intent" (WtB p. $\left.5^{2}\right)$.

23 Platon wird in $W t B$ nur einmal erwähnt, Aristoteles übrigens gar nicht. In VRE nennt James Platon und auch Aristoteles (einmal kritisch), nicht jedoch Plotin, Thomas von Aquin oder Nikolaus Cusanus; S. Kierkegaard als mögliche Quelle mancher Gedanken wird ebenfalls selten genannt (vgl. Thörner 2011, p. 219 / 4).

$24 W t B$ p. 52; vgl. ähnlich p. 44: „If there be a divine Spirit of the universe, nature, such as we know her, cannot possibly be its ultimate word to man. Either there is no Spirit revealed in nature, or else it is inadequately revealed there; and (as all the higher religions have assumed) what we call visible nature, or this world, must be but a veil and surface-show whose full meaning resides in a supplementary unseen or other world."

25 Auch Ricken sieht das Problem: Die Religion sage, die besten Dinge seien die ewigen (WtB p. 25). Verträgt sich das dynamische Welt- und Menschenbild mit derartigen Anleihen beim Platonismus? „James entwickelt, unter ausdrücklicher Berufung auf das Symposion, eine platonische Ontologie. Für ihn gibt es keine Tatsachen ohne Werte. Das gesamte Universum konkreter Gegenstände erhält seine Bedeutung von einem weiteren und 
Aus theologischer Sicht müsste zudem wohl problematisiert werden, dass James die Ansicht, die physische Welt sei in einem absoluten und endgültigen Sinn das Ziel göttlicher Gründung, sehr frühen Religionen wie „den primitivsten Juden" zuschreibt. ${ }^{26}$ Außerdem erscheint es mir mindestens als unglücklich, den positiven Gegenbegriff „supernaturalistisch“ zu nennen. Die abgelehnte Gottesvorstellung der natürlichen Theologie bezeichnet James als „Idol des Geistes der Natur" und "monistischen Aberglauben“27 und sieht das Ideologische in der Verhärtung dieses Glaubens zu einer rationalistischen Metaphysik, auch „scholastischer Absolutismus“ genannt. Der scholastische Absolutist meint, einen "infallible intellect with its objective certitudes“ $\mathrm{zu}$ haben, was aber der Empirist nicht annehmen könne (WtB p. 30). Das ist der eine Gegner seines Verständnisses von legitimer Religiosität. Er definiert demgegenüber Religion

in the supernaturalistic sense, as declaring that the so-called order of nature, which constitutes this world's experience, is only one portion of the total universe, and that there stretches beyond this visible world an unseen world of which we now know nothing positive, but in its relation to which the true significance of our present mundane life consists (WtB p. 51).

Das Relat der unsichtbaren Welt im Menschen ist seine „passional“ oder „willing nature", klassisch formuliert: seine Strebensnatur, welche die existenzielle Bedeutung jenseits der wahrgenommenen Welt sucht. Bevor wir diesem Gedanken bei James folgen, dass die Frage nach dem Sinn des Lebens das

höheren Universum abstrakter Ideen, z. B. des Guten, der Schönheit, der Gerechtigkeit. Sie bilden den Hintergrund für alle Tatsachen; jedes einzelne Ding hat an ihnen teil“ (Ricken 2003, p. 65). VRE Vorlesung II behauptet die Bestimmbarkeit des menschlichen Geistes von Ideen durch eine Art Wahrnehmung, ein Realitätsgefühl wie es Liebende von einander haben. Das ist „Mystizismus“ im Gegensatz zu „Rationalismus“, der leugnet, dass wir Zugang zu einer Wirklichkeit haben, die sich nicht sprachlich artikulieren und begründen lässt (VRE pp. 72-74). Der Verstand artikuliert, interpretiert, kritisiert auch aber er generiert nicht den Bezug zum Göttlichen. Eine andere Ontologie zeigt sich in der Schlussvorlesung VRE Kap. Xx: „An die Stelle der abstrakten platonischen Objekte [...] ist jetzt das ,Mehr' getreten, das ich als eine neuplatonisch zu verstehende Tiefendimension des Bewußtseins oder Subjekts deute“ (Ricken 2003, p. 69).

26 "The notion that this physical world [...] is absolutely and ultimately the divinely aimedat and established thing, is one which we find only in very early religions, such as that of the most primitive Jews" ("Is Life worth Living“, in $W t B$ p. $5^{2}$ ). „an idol of the spirit of nature“, „monistic superstition“ (WtB p. 46). 
natürliche Universum spirituell aufbricht, untersuchen wir die zweite Gegnerschaft im wissenschaftlichen Absolutismus (WtB p. 25), der religionsskeptisch ist aufgrund einer Wissenschaftsgläubigkeit und eines Intellektualismus, der ebenfalls meint, sich objektiver Evidenz versichern zu können.

Der allein wissenschaftsgläubige Mensch mag zugeben, dass es ein Bedürfnis oder einen Wunsch zu glauben geben kann, erklärt ihn aber für intellektuell nicht verantwortbar, ja verwerflich. Es sei dumm, zu glauben, weil man es wünscht. ${ }^{28}$ In $W t B$ steht für diese Position vor allem William Kingdon Clifford, den James zitiert: Einen Glauben anzunehmen „on insufficient evidence [...] is sinful because it is stolen in defiance of our duty to mankind [...]. It is wrong always, everywhere, and for every one, to believe anything upon insufficient evidence.“29 Demzufolge müsse man sich eines religiösen Glaubens enthalten. ${ }^{30}$

Was James dagegen setzt, ordne ich als eine Klärung und drei Argumente. Wenn der Szientismus die „willing nature“, die strebende (auch spirituelle) Natur des Menschen negiert, sehe er diese gar nicht richtig, nicht in ihrer das ganze menschliche Leben prägenden phänomenalen Breite. James versteht unter "willing nature" nicht nur bewusstes Wollen, sondern allgemein unausweichliche und gewohnte Annahmen. „I mean all such factors of belief as fear and hope, prejudice and passion, imitation and partisanship, the circumpressure of our caste and set“ (WtB p. 9). Das intellektuelle Klima übe tatsächlich eine Autorität aus (letztlich durch "prestige of the opinions“) in seiner akademischen Welt eben eine religionsskeptische. Über die nichtrationale Basis entsprechender Wissenschaftskultur solle man sich nicht täuschen.

Das erste Argument in meiner Systematisierung besagt: Die Position Cliffords leide an dem Selbstmissverständnis, dass ihre objektive Wissenschaftlichkeit frei wäre von nicht-rationalen Motiven. Das zeige sich zunächst

28 „believing by our volition seems [...] simply silly“ (WtB p. 7).

29 Zitat in $W t B$ p. 8 ohne Quellenangabe. Vgl. die Charakterisierung der Position in $W t B$ p. 129. Der Aufsatz findet sich in Clifford 1999, pp. 70-96.

30 Im Rahmen dieses philosophiegeschichtlich konzipierten Beitrags kann auf die weitergeführte sachliche Auseinandersetzung zu „Clifford's principle “und James' Gegenargumente nur hingewiesen werden: Kitcher 2004 und die in Kitcher 2004, p. 135 / 41 genannten und diskutierten Positionen von Peter van Inwagen und Allen Wood. 
in der Entscheidung zwischen zwei Imperativen, wo diese nicht zugleich befolgt werden können: „Suche die Wahrheit!“ und „Vermeide den Irrtum!“ Cliffords Präferenz für den zweiten Imperativ, der religiösen Glauben verbietet, stamme aus Angst vor Irrtum, also aus ebenso irrationalen Motiven wie der entgegengesetzte Wille zum Glauben (WtB p. 17-19). Folglich kann der Agnostiker in dieser Hinsicht gegenüber dem Gläubigen keine epistemische Superiorität in Anspruch nehmen. Positivisten vergöttlichen letztlich eben die Wissenschaft: „Certain of our positivists keep chiming to us, that, amid the wreck of every other god and idol, one divinity still stands upright, - that his name is Scientific Truth, and that he has but one commandment [...] Thou shalt not be a theist" (WtB p. 131).

Zweitens mache sich der Intellektualismus ein viel zu einfaches Bild von der Genese unserer Überzeugungen. Wohl gebe es in der Wissenschaft einige triviale und oft tote Optionen, in solchen Fällen sei eine skeptische Unentschiedenheit weise. Aber schon im Forschungsverlauf selbst schade solche Indifferenz (WtB p. 21) - in dem, was die spätere Wissenschaftstheorie "context of discovery" oder Entdeckungszusammenhang nennt. Ohne Leidenschaft, ohne eine Art Glauben an eine Hypothese könne sie nicht weit genug entwickelt werden, um in den Kampf um die Wahrheit geschickt zu werden. Und auch der Trieb der konkurrierenden Wissenschaftler, eine Hypothese zu falsifizieren, ist in Rechnung zu stellen. Das Streben nach Erkenntnis müsse wohl ausbalanciert werden von Nervosität angesichts der Möglichkeit, sich zu täuschen (und falsifiziert zu werden). „Science has organized this nervousness into a regular technique, her so - called method of verification“ (WtB p. 21). Gemäß dem zweiten Argument versteht also die szientistische Position schon ihren eigenen Wissenschaftsbetrieb falsch, weil sie in ihrem Intellektualismus den Menschen einseitig sieht.

Drittens aber verfehlt sie wesentliche Unterscheidungen zwischen diversen menschlichen Vollzügen, insbesondere zwischen interpersonalen und kooperativen Phänomenen, moralischen und religiösen Entscheidungen einerseits und empirisch entscheidbaren Fragen auf der anderen Seite (WtB pp. 22-25). Pascals Gründe des Herzens seien aber nötig für Fragen, die Werte implizieren; das sind natürlich moralische Fragen und Entscheidungen in zwischenmenschlichen Beziehungen. Solche werden wesentlich mitbestimmt von "trust and expectation“ (WtB p. 23), diese kreieren geradezu die Verifikation. James wendet sich gegen die Logik der wissenschaftlichen Absolutisten in Belangen der Moral und des Miteinanders, aber er bezieht dann vor allem religiöse Fragen, als bedeutsame und unvermeidliche Optionen, mit ein. Auch für sie gilt: „In truths dependent on our personal action, then, faith based on desire is certainly a lawful and possibly an indispensable thing." (WtB 
p. 25) Die Struktur wesentlicher menschlicher Erfahrungen weist Vertrauen und Wagnis als eine Vorbedingung auf. ${ }^{31}$

Dies gilt aufgrund von James' Anthropologie, Entscheidungs- und Erkenntnistheorie unabhängig davon, ob auch der religiöse Bezug als personaler gefasst wird. Er nennt den Buddhismus und R. W. Emersons Transzendentalismus als Formen von Religion ohne personale Gottesvorstellung ${ }^{32}$ und stellt einfach fest, dass in „unseren Religionen“, wohl die monotheistischen meinend, eine personale Form vorliege. Für diese ist es nur noch plausibler, dass der sich enthaltende Skeptiker in seiner "snarling logicality“, also in etwa knurrender logischer Rechthaberei, keine religiöse Erfahrung wird machen können. Wenn James den personal-dialogischen Aspekt des Gottesglaubens mit den Verifikationsbedingungen für Liebesbeziehungen und kooperative zwischenmenschliche Verhältnisse parallelisiert, eröffnet er den Raum für religiöse Erfahrung. Anders gesagt: Wenn man sich nicht auf epistemische Kriterien für die Rechtfertigung von Glauben einigen kann, müssen die Konsequenzen von skeptischen und leichtgläubigen Haltungen verglichen werden. In VRE wird James eben die Folgen des Glaubens entfalten. ${ }^{33}$

Der agnostische Positivismus erkenne theoretisch und in abstracto durchaus die Stückhaftigkeit unseres Wissens an - aber eine daraus gefolgerte Neutralität in religiösen Fragen sei dem Menschen innerlich schwierig und äußerlich undurchführbar. Die menschliche Beziehung zur Alternative "Glaube oder Unglaube“ ist praktisch und vital, beides hat Konsequenzen im Verhalten. Ja, das Zweifeln kann sich nur ausdrücken in einem Verhalten, als ob das Bezweifelte nicht wäre. Wer denkt, nicht glauben zu dürfen, dass die

31 „Nur durch die Wahl, von unserer Natur erfordert, können wir, bevor wir die Evidenz klar eingesehen haben, die Evidenz erreichen" (McCool 1990, p. 770).

32 Siehe z. B. VRE p. 31. Gegenüber seinen Zuhörern vom unitarischen Institut indessen unterstreicht James 1881 die Bedeutung des personalen Theismus: „it changes the dead blank it of the world into a living thou, with whom the whole man may have dealings" („Reflex Action“, in WtB p. 127).

33 Auf dieser Linie argumentiert auch Kitcher für eine Lesart der VRE von WtB her, als Ausarbeitung der systematischen und dominanten Effekte religiösen Glaubens (vgl. „A Pragmatist's Progress“, pp. 123-126). Zusammengefasst: „It is agreed that we have no way of coming to know religious claims, and the question to be decided is whether it is permissible to believe such claims on the basis of evidence that would not suffice for knowledge. Belief is permissible provided that there is a way of embedding individuals' commitments to religion in a framework of social practices that will produce overall consequences at least as good as those of any alternative that forbids such commitments. The beliefs induced by religious experiences have as a dominant consequence their alleviation of a predicament that the most reflective human beings share, and, indeed, the fruits of endorsing religious experiences typically consists in the transformation of the individual's life in productive ways." (Kitcher 2004, pp. 124 et seq.). 
Welt göttlich sei, kann dies nur so ausgestalten, dass er sich in irreligiöser Weise verhält. In manchen kritischen Entscheidungen gibt es keine konsistente Neutralität (WtB pp. 54 et seq.). Darum formuliert James das Gesetz, dass die strebende Natur entscheidungsbefugt ist, wenn eine Option genuin und intellektuell unentscheidbar ist (und aus dem Kontext ist klar, dass sie auch lebendig und bedeutsam sein muss). Denn Indifferenz riskiere ebenso wie eine falsche Entscheidung, die Wahrheit zu verfehlen. ${ }^{34}$

James konstatiert eine strukturelle Parallele von szientistischem und religiösem Glauben: Er geht ausdrücklich von einem religiösen Bedürfnis des Menschen aus und verteidigt es durch Vergleich mit dem Bedürfnis der Wissenschaftler, uniforme Kausalgesetze anzunehmen. ${ }^{35}$ Er spricht sogar von der „religion of exclusive scientificism“ (WtB p. 132). Der Positivist wird einwenden: Das Leben als ob es die weitere spirituelle Welt gäbe, legitim insoweit es sie vielleicht („maybe“) gibt, sei doch mit einer wissenschaftlichen Haltung unvereinbar. James antwortet, „the 'scientific' life itself has much to do with maybes, and human life at large has everything to do with them" (WtB pp. $5^{8}$ et seq.).

Schließlich reformiert James Pascals Wette ${ }^{36}$ - der komparative Vorteil des Glaubens werde nicht erst nach dem Tod, sondern schon während dieses Lebens eingelöst. Denn Skeptizismus in Form von Agnostizismus entkommt nicht der Entscheidung - der "faith-vetoer“, der sich den Glauben verbietet, verliert das mögliche Gut des Glaubens ebenso wie der entschiedene Atheist. Durch diese De-Eschatologisierung des Wettarguments wird nun der Raum und die entscheidende Relevanz religiöser Erfahrung zugänglich. Ein Insistieren auf rationaler Absicherung, bevor geglaubt würde, das eben so den Zugang zu möglichen Wahrheiten verschließt, ist selbst irrational. ${ }^{37}$ Es verschließt auch die Lebendigkeit menschlichen Strebens, verstopft das Herz,

34 Siehe $W t B$ p. 11.

35 „But the inner need of believing that this world of nature is a sign of something more spiritual and eternal than itself is just as strong and authoritative in those who feel it, as the inner need of uniform laws of causation ever can be in a professionally scientific head.“ („Is Life worth Living“, in WtB p. 56).

36 Pascal: Pensées fr. 233. Auf den ersten Blick hatte James Pascals Wettargument kritisiert, doch tatsächlich argumentieren beide in paralleler Weise: „Wenn man aber die allgemeine Form von Pascals Argumentation betrachtet, der zufolge zwei Risiken nicht nur nach ihren Wahrscheinlichkeiten, sondern auch nach ihren voraussichtlichen »Einbußen« gegeneinander abgewogen werden sollten, dann scheint James selbst einen vergleichbaren Standpunkt zu vertreten." (Taylor 2013, p. 47). Überdies schränkt Pascal selbst den möglichen Gewinn oder Verlust der Glaubensentscheidung nicht ganz auf eine postmortale Existenz ein.

Vgl. WtB p. 28. 
die Instinkte und den Mut - es ist ein „command that we shall put a stopper on our hearts, instincts, and courage" (WtB p. 29).

Wir kommen zu dem Argument, das der Struktur der Legitimierung religiöser Erfahrung bei James m.E. zugrunde liegt und die mehrfache Frontstellung verständlich macht. Was haben scholastischer und wissenschaftlicher Absolutismus gemeinsam? Was lehnt James ab sowohl in der natürlichen Theologie, wie er sie kennt, als auch im szientistischen Agnostizismus? Beides sind Formen des Dogmatismus, beide pflegen die Ideale der objektiven Evidenz und der Sicherheit des Erkennens, was für den radikalen Empiristen illusionär ist.

Sicherheitsstreben darf als ein Grundmotiv modernen Denkens gelten hier müssen Hinweise genügen: Nach René Descartes darf nur das als klar und unbezweifelbar oder sicher und evident Erkannte für wahr gelten. ${ }^{38}$ Und bekanntlich fand Descartes in der Selbstgewissheit des Bewusstseins den sicheren, unerschütterlichen archimedischen Punkt.. ${ }^{39}$ John Locke fragt am Beginn von An Essay Concerning Human Understanding, welchen Grad der Gewissheit Erkenntnis erreichen kann ${ }^{40}$ und ordnet im IV. Buch die Erkenntnisweisen nach dem Grad der Gewissheit. Für David Hume ist die Quelle sicherer Erkenntnis „experience“, er empfiehlt seine Philosophie als einziges sicheres Fundament. ${ }^{41}$ In der Gründungsschrift neuzeitlicher politischer Philosophie, dem Leviathan von Thomas Hobbes, findet sich im zentralen Kap. XVII allein auf den ersten Abschnitten sechs Mal das Schlüsselwort

38 Vgl. dazu die erste Regel im Discours de la méthode, Descartes 2001, p. 39. In der Vorrede an die Doktoren zu den Meditationes de prima philosophia bezeichnet Descartes die vorgelegten Gründe als gewisseste und evidenteste Beweise („pro certissimis et evidentissimis demonstrationibus"); Descartes 2011, p. 20. Die Vorrede an den Leser verspricht gewisse und evidente Wahrheitserkenntnis („certam et evidentem cognitionem veritatis"); Descartes 2011, p. 36.

39 Descartes 2011, p. 68: „certum [...] inconcussum“. „Der Begriff der G. [= Gewissheit] erhält nunmehr eine grundsätzlich neue Prägung und seine für das neuzeitliche Denken kennzeichnende Relevanz. Die Frage nach der G. wird zur Frage nach den Möglichkeiten und Grenzen menschlicher Erkenntnis zugespitzt und der Frage nach der Wahrheit vorangestellt“ (Halbfass 1974, Sp. 593).

$40 \quad$ Locke 1979, I,1 § 2, pp. 43-45.

41 Hume 1739. In der Introduction verspricht Hume „a compleat system of the sciences, built on a foundation almost entirely new, and the only one upon which they can stand with any security“ (Hume 1739, p. Xx). 
„secure“ bzw. „security“.42 James kennt den cartesischen Zweifel, in dem man sich nur des eigenen Bewusstseins sicher sein kann, aber will ihn nicht überwinden um den Preis, das Gegebensein der Dinge zu negieren. Insoweit Sicherheitsstreben ein Grundmotiv modernen Denkens ist, sieht James die Zeit gekommen, die von konkurrierenden Gesellschaften angebotenen geistigen Versicherungsverträge alle zu kündigen. Absolutisten oder Dogmatisten und Empiristen glauben zwar beide, dass der Mensch die Wahrheit erkennen kann, aber Empiristen zweifeln daran, dass man sich dessen sicher sein könne. Die Philosophiegeschichte zeige eine absolutistische Tendenz "in the conviction felt by each successive school or system that by it bottom-certitude had been attained" (WtB p.12). Als Beispiel zitiert James die absolutistische Überzeugung der scholastischen Orthodoxie von der objektiven Evidenz. Aber auch der Szientismus habe einen solchen unkritischen Glauben (!) an objektive Evidenz, ja letztlich gelte: „we are all such absolutists by instinct“ (WtB p. 13). Der „complete empiricist“ in erkenntnistheoretischer Hinsicht fordert hingegen, sich von der Illusion zu befreien, dass „indefectibly certain truth“ zu finden wäre (WtB p. 14). Seine Formulierungen zeigen, dass James Objektivität und Sicherheit zusammen sieht. ${ }^{43}$ Nach einer langen Liste an Beispielen für behauptete sichere Evidenzen in kontradiktorischen Positionen der Philosophiegeschichte versäumt James nicht, darauf hinzuweisen, dass "the most striking practical application to life of the doctrine of objective certitude has been the conscientious labors of the Holy Office of the Inquisition" (WtB pp. 16 et seq.).

Das Sicherheitsstreben der theoretischen Weltinterpretation werde allerdings immer wieder aufgebrochen: Dogmatischer Theismus durch das sinnliche Aufnahmevermögen und die widerspenstigen Fakten, dogmatischer Naturalismus durch die Strebensnatur des Menschen. ${ }^{44}$ Wenn James klarstellt, ,as empiricists we give up the doctrine of objective certitude, we do not thereby give up the quest or hope of truth itself" (WtB p. 17), reformuliere ich das als Versuch, den Bezug zur Wahrheit von der pseudoobjektiven Sicherheit und der damit gegebenen geistigen Sklerose zu befreien.

Wohl lasse sich die naturalistische Reduktion aufgrund einer dem Menschen angeborenen naturalistischen und materialistischen Tendenz verstehen. "Of this sort of mind the entity called 'science' is the idol" (WtB

\footnotetext{
42 Hobbes 1994, pp. 106-109.

43 "Objective evidence and certitude are doubtless very fine ideals to play with" (WtB p. 14).

44 Der frühe Vortrag „Reflex Action and Theism“ (WtB pp. 111-143) erläutert die minimaltheistische Position eines rationalen Equilibriums - noch ohne der mystischen Erfahrung eine erkenntnisbegründende Rolle zuzumessen.
} 
p. 52). Demnach ist Szientismus eine Art Götzendienst, verständlich aufgrund der beeindruckenden Erfolge empirischer Wissenschaft und auch der Moral vorbildlicher Forscher. ${ }^{45}$ James skizziert den Fortschrittsoptimismus einiger Kollegen, dass die Fundamente der Wahrheit wissenschaftlich erforscht seien und nur noch Details aufzufüllen wären. Aber es sei „barbarisch“, die Wissenschaft nach der kurzen Dauer von kaum 300 Jahren (seit Galileo) für so weit gediehen zu halten, während doch ständig neue Konzepte und neue Probleme gefunden werden. Er nennt es „mushroom knowledge“ - über Nacht gekommen und maßlos überschätzt. Dagegen möchte er die Proportion von wissenschaftlichem Wissen und Unwissen zurecht rücken. ${ }^{46}$

Wir sehen James' Bestreben, die phänomenal vielgestaltige und mehrdimensionale Welt zu öffnen oder offen zu halten gegenüber einem wissenschaftlichen Weltbild, das sich für beinahe abgeschlossen hält und damit verschließt gegen das immer wieder überraschende Andere, Plurale und Neue - schon im Bereich des prinzipiell empirisch Erforschbaren, aber erst Recht im transzendenten Bereich. Aus ähnlicher Sorge über den Verlust der Offenheit und Vielfalt der Welt kritisiert er den Hegelschen Idealismus, so wie er ihn versteht, als Stilllegung von Pluralität und Dynamik im monistischen Absoluten. ${ }^{47}$ Er hinterfragt den intellektuellen Despotismus gegenüber den Grenzen des Gewussten mit der polemischen Frage: „By what insatiate conceit and lust of intellectual despotism do I arrogate the right to know their secrets, and from my philosophic throne to play the only airs they shall march to, as if I were the Lord's anointed?" (WtB p. 271).

James beharrt auf dem unvordenklichen Gegebensein der Dinge und fordert Demut und Dankbarkeit dafür: „Data! gifts! something to be thankful for! It is a gift that we can approach things at all.“48 In "Reflex Action and Theism“

45 "Science has made such glorious leaps in the last three hundred years, and extended our knowledge of nature so enormously both in general and in detail; men of science, moreover, have as a class displayed such admirable virtues, - that it is no wonder if the worshippers of science lose their head" (WtB p. 53).

46 „No! Our science is a drop, our ignorance a sea“ (WtB p. 54).

47 Siehe die Verteidigung des „pluralist-empiricist point of view“ (XIII) gegen eine Hegelsche Dialektik der Begriffe allein im Ix. Essay („On some Hegelisms“, in WtB pp. 263-298). Auch hier lässt sich am Text zeigen, dass James die Grundthese von der Identität des Realen mit dem Idealen als Hybris (p. 272, vgl. 264), letztlich als Versicherungssystem gegen Heterogenität und Vielfalt ansieht. Es ist für ihn eine moralische Einstellung („moral view“), die pluralen Teile des Universums nicht aus einer einzigen, absoluten Perspektive kontrollieren zu wollen, sondern ihnen Freiheit zu lassen.

$48 W t B$ p. 271. Vgl. seine Charakterisierung der idealistischen Philosophie als unfähig, eine unkontrollierbare Wirklichkeit zu akzeptieren und sich Dinge und Möglichkeiten geben zu lassen: „Data! facts that say, 'Hands off, till we are given'! possibilities we can't control! 
spricht James mehrfach vom Sein, das als Gegebensein anzunehmen ist und etwas Heiliges an sich hat: „In every being that is real there is something external to, and sacred from, the grasp of every other."49 Selbst wenn es dem theoretischen Zugriff des Geistes je gelänge, die vielfältige Realität in einer einheitlichen Theorie zu begreifen, würde die Grundfrage immer noch offen sein: Wieso ist überhaupt etwas und nicht vielmehr nichts? „Why was there anything but nonentity"? 50

Glauben in einem allgemeinen Sinn ist ein Grundverhältnis des denkenden Lebewesens. "We cannot live or think at all without some degree of faith" (WtB p. 95). Wir müssen Gegebenes annehmen und diesbezügliche Hypothesen annehmen. James entwickelt im Folgenden eine Kurzform des wissenschaftstheoretischen Falsifikationismus: Manche Annahmen werden in fünf Minuten widerlegt, andere beschäftigen Generationen von Forschern, die alle so experimentieren, als wären die Hypothesen wahr - und warten auf ihre Falsifikation. „The longer disappointment is delayed, the stronger grows his faith in his theory" (WtB p. 95). Gläubige Annahmen über Gott nun, über Unsterblichkeit, absolute Moral und freien Willen sind wie alle Hypothesen immer bezweifelbar, aber die Gläubigen erachten die Plausibilität für stark genug, um danach zu handeln und sie zu bewähren, während eine endgültige Bestätigung oder Widerlegung bis zum Jüngsten Tag warten möge. Während James von der Berechtigung spricht, den eigenen religiösen Bedürfnissen zu trauen (trusting), ist ihm wichtig, klarzustellen, dass dies keine Erlaubnis mit sich führe, die unsichtbare Welt im Detail zu definieren und Menschen mit anderem Glauben oder Vertrauen zu exkommunizieren. „Our faculties of belief were not primarily given us to make orthodoxies and heresies withal; they were given us to live by" (WtB p. 56).

Wir sehen hier eine weitgreifende Kritik des Sicherheitsstrebens, das für die empirische Wissenschaft hingehen mag (und sei es als methodische Illusion), für die Lebenspraxis aber untauglich, ja schädlich ist. Mit einem Zitat von Fitz James Stephen plädiert James am Ende von WtB (p. 31) für den Mut ,to take a leap in the dark“.

a banquet of which we merely share! Heavens, this is intolerable; such a world is no world for a philosopher to have to do with. He must have all or nothing. If the world cannot be rational in my sense, in the sense of unconditional surrender, I refuse to grant that it is rational at all." (WtB p. 272).

$W t B$ p. 141 - auf derselben Seite spricht er vom "pulse of Being“ (groß geschrieben). Vgl. p. 143, Charles Renouvier zustimmend: ,this very empirical element in theism, its confession of an ultimate opacity in things, of a dimension of being which escapes our theoretic control". 
Was wird nun anders durch das Wagnis eines religiösen Glaubens? Wodurch wird ein solcher womöglich verifiziert? Abschließend nenne ich nur kurz zwei Bereiche, die ethische Motivation und die Mystik.

1. Ethische Anstrengung wird durch Glauben in unüberbietbarer Weise motiviert.

Our attitude towards concrete evils is entirely different in a world where we believe there are none but finite demanders, from what it is in one where we joyously face tragedy for an infinite demanders' sake. Every sort of energy and endurance, of courage and capacity for handling life's evils, is set free in those who have religious faith (WtB p. 213).

Ein göttlicher Gesetzgeber ist aus ethischer Sicht $\mathrm{zu}$ postulieren. Eine politische Ordnung, welche einen friedlichen und fruchtbaren Wettstreit von Glaubensüberzeugungen und entsprechenden Lebenspraxen ermöglicht, wird von James einfach vorausgesetzt. ${ }^{51}$ Aus heutiger Sicht, der das destruktive Potential von Religion viel bewusster ist, wäre zu fragen, ob James nicht die Konsequenzen zu einseitig sieht und damit seine bewusste Ausgrenzung der sozialen und ekklesialen Dimension von Religion mitsamt der von Glaubensgemeinschaften entwickelten Theologien eine schädliche Perspektivverengung verursacht. Stellt das nicht eine Form von „healthy-mindedness“ dar, die genauso als selektive Wahrnehmung und als philosophisch unredlich $\mathrm{zu}$ charakterisieren ist, wie James es in VRE (Vorlesungen IV-V) tut?

2. In der Praxis als Bewährung des Glaubens und in mystischer Erfahrung kann das Göttliche in die Welt kommen, wo sie nicht in rationalistischer Weise als abgeschlossen betrachtet wird. In $V R E$ entfaltet James die Wirkungen dessen, was er in $W t B$ legitimiert, in der Vielfalt religiöser Erfahrung auf den Stufen eines religiösen Gefühls, einer ausdrücklichen Bekehrung und der Heiligkeit. Auch mystische Zustände sind „Hypothesen“ (VRE p. 428). Religiöse Erfahrung bewahrheitet Glauben (in verschiedenen Traditionen und Interpretationssystemen!) durch seine Evidenz, Konsistenz und erfahrene Passung zum emotionalen Leben. Mystische Erfahrungen brechen das rationalistische und sensualistische Bewusstsein auf, führen aus dessen Enge in befreiende Weite:

$5^{1}$ John Dewey widmete sich den politisch-pädagogischen Folgerungen viel mehr als James, vgl. dazu Dewey 1916. 
They break down the authority of the non-mystical or rationalistic consciousness, based upon the understanding and the senses alone. They show it to be only one kind of consciousness. They open out the possibility of other orders of truth, in which, so far as anything in us vitally responds to them, we may freely continue to have faith (VRE p. 423).

Mystische Erfahrung eröffnet dann auch dem Nicht-Mystiker neue Hypothesen in Bezug auf Leben und Welt; diese müssen wiederum in der Praxis geprüft werden. „Sie müssen sich ausweisen durch ihre hermeneutische Kraft, die neue und umfassendere Sicht seines Lebens, und dadurch, daß das Ideal, von dem sie sprechen, dem mystischen Kern in ihm antwortet und so neue Kräfte entbindet.“52 Wie Hans Julius Schneider betont, schließt das ein, dass die Übel des Lebens wahrgenommen werden. Die leidhafte Seite wird durch Erfahrungen von Machtlosigkeit und durch das Aufgeben von Bemächtigungsversuchen integrierbar, insoweit es dem Glaubenden widerfährt, dass er trotz seines Loslassens nicht untergeht, dass er aufgehoben wird. ${ }^{53}$ James beruft sich auf die Erfahrung, nach einem "Schiffbruch" aufgehoben und getragen zu werden (vgl. VRE p. 508). Denn es geht nicht um ein Wahrnehmen neuer Tatsachen, sondern um eine veränderte Sichtweise auf das Ganze der Wirklichkeit und des Lebens. „Religion [...] is a man's total reaction upon life“ (VRE p. 35).

Die neue Sicherheit wird erreicht durch Hingabe, Selbstübergabe geschehe sie plötzlich, in einer mystischen Bekehrungserfahrung (siehe VRE Kap. IX und X) - oder Schritt für Schritt unter größerem Einsatz des Willens, dennoch nicht ohne Selbstübergabe. ${ }^{4}$ Auch mystisch Unbegabte (wie James selbst) können dem mystischen Keim in ihnen Raum zum Wachsen, Reifen und Fruchtbringen geben.

Ist der Anspruch des dogmatischen Erkenntnisstrebens, seiner Sicherungsprätentionen und entsprechender Methoden einmal eingegrenzt oder aufgebrochen, kann Unsicherheit angenommen werden, geschieht Loslassen bis hin zu einem heilsamen Sich-aufgeben. ${ }^{55}$

There is a state of mind, known to religious men, but to no others, in which the will to assert ourselves and hold our own has been displaced by a willingness to close our mouths and be as nothing in the floods and waterspouts of God. In this state of mind, what we most dreaded has

$52 \quad$ Ricken 2003, p. 76.

53 Vgl. Schneider 2007, pp. 178-180.

54 Siehe Ricken 2003, p. 70.

55 Vgl. die subjektive Seite der insecuritas humana bei Wust 1986, pp. 31-59. 
become the habituation of our safety, and the hour of our moral death has turned into our spiritual birthday. ${ }^{56}$

Erfahrbar wird so eine Zeit glücklicher Entspannung und ewiger Gegenwart („eternal present ${ }^{457}$ ). Der Friede des Geistes wird nicht erreicht durch Beantwortung der letzten existenziellen Fragen auf intellektueller Ebene, sondern in dem, was James mystisch nennt oder auch Ekstase.

To religious persons of every shade of doctrine moments come when the world, as it is, seems so divinely orderly, and the acceptance of it by the heart so rapturously complete, that intellectual questions vanish [...]. Ontological emotion so fills the soul that ontological speculation can no longer overlap it and put her girdle of interrogation-marks round existence. $^{58}$

Die interessante Formulierung und Behauptung ontologischer Emotionen als Gegenbegriff zu ontologischer Spekulation setzt eine Psychologie voraus, die grundlegend zwischen Sentiment und Rationalität unterscheidet. Im Rückgriff auf geschichtliche Positionen europäischer Philosophie hätte James hier an den Unterschied von nous und dianoia oder intellectus und ratio anknüpfen können, um über die Entgegensetzung von "speculation“ und „emotion“, von "rational" und "mystisch“, von Kopf und Herz hinauszukommen.

An dieser Stelle wäre die mystische Erfahrung nach James' VRE weiter zu entfalten, was allerdings hier nicht ansteht. Meine Aufgabe sah ich darin, zu zeigen, wogegen und wie James den Raum für religiöse Erfahrung eröffnet, in der nordamerikanischen Wissenschaftskultur seiner Zeit, die er für intellektualistisch hielt, auf Kosten einer Anerkenntnis der (ethisch und religiös) strebenden Natur des Menschen, und für ebenso dogmatisch wie die rationalistische Metaphysik. Gegen beide geistigen Absolutismen argumentierte er für eine erfahrbare und pragmatisch verifizierbare religiöse Wahrheit - was zur Voraussetzung hat, das Ende eines typisch modernen Sicherheitsstrebens mit zu vollziehen.

\footnotetext{
$56 \quad$ VRE p. 47.

57 VRE p. 47. Die Erfahrung göttlicher Gegenwart beruht, wie James allgemeiner formuliert, in "that curious sense of the whole residual cosmos as an everlasting presence“, der dann als vertraut oder fremd, furchtbar oder komisch, liebens- oder hassenswert erscheinen kann (VRE p. 42).

$5^{8}$ „The Sentiment of Rationality“, in $W t B$ p. 74.
} 


\section{Biography}

Markus Riedenauer, now Professor of Philosophy in the Theology Faculty of the Catholic University of Eichstätt-Ingolstadt, studied philosophy, catholic theology and history at universities in Munich, Rome and Vienna. His doctorate in philosophy from the Universität Wien was followed by his Habilitation at the Goethe-Universität Frankfurt.

His research areas include practical philosophy, particularly in the Aristotelian tradition, philosophical practice, medieval and early modern philosophy, philosophical theology and philosophy of religion.

\section{Bibliography}

Clifford, William K.: The Ethics of Belief and Other Essays, Amherst NY: Prometheus Books 1999.

Descartes, René: Discours de la Méthode/Bericht über die Methode. Französisch/ Deutsch. Stuttgart: Reclam 2001 (translated by Holger Ostwald).

Descartes, René: Meditationen. Dreisprachige Parallelausgabe Latein - Französisch Deutsch. Ed. by Andreas Schmidt. Göttingen: Vandenhoeck \& Ruprecht ${ }^{2} 2011$.

Dewey, John: Democracy and Education. An Introduction to the Philosophy of Education. New York: The Macmillan Company 1916.

Habermas, Jürgen: „Religion in der Öffentlichkeit. Kognitive Voraussetzungen für den ,öffentlichen Vernunftgebrauch“ religiöser und säkularer Bürger“, in: id.: Zwischen Naturalismus und Religion. Philosophische Aufsätze. Frankfurt am Main: Suhrkamp 2005, pp. 119-154.

Haeffner, Gerd: „Erfahrung - Lebenserfahrung - religiöse Erfahrung. Versuch einer Begriffsklärung“, in: Ricken, Friedo (ed.): Religiöse Erfahrung. Ein interdisziplinärer Klärungsversuch. Stuttgart: Kohlhammer 2004, pp. 15-39.

Halbfass, Wilhelm: „Gewissheit“, in: Historisches Wörterbuch der Philosophie (HWPh), Bd. 3. Basel (et al.): Schwabe 1974, pp. 592-594.

Hobbes, Thomas: Leviathan. With selected variants from the Latin edition of 1668. Ed. by Edwin Curley. Indianapolis, Cambridge: Hackett Publishing Company 1994. Hume, David: A Treatise of Human Nature. London: printed for John Noon, 1739.

James, William: The Letters of William James. Ed. by Henry James. Bd. II. Boston: The Atlantic Monthly Press 1920.

James, William: The Will to Believe and other essays in popular philosophy. New York/ London: Longmans, Green, and Co 1927 (cited as WtB).

James, William: The Varieties of Religious Experience. A Study in Human Nature. London/ New York/Toronto: Longmans, Green, and Co 1929 (cited as VRE). 
James, William: Die Vielfalt religiöser Erfahrung. Eine Studie über die menschliche Natur. Berlin: Verlag der Weltreligionen 2014 (cited as Vielfalt).

Kitcher, Philip: „A Pragmatist's Progress: The Varieties of James' Strategies for Defending Religion“, in: Wayne Proudfoot (ed.): William James and a Science of Religions. Reexperiencing The Varieties of Religious Experience. New York: Columbia University press 2004 .

Krämer, Felicitas: Erfahrungsvielfalt und Wirklichkeit. Zu William James' Realitätsverständnis. Göttingen: Vandenhoeck \& Ruprecht 2006.

Locke, John: An Essay Concerning Human Understanding. New York: Oxford University Press 1979 .

McCool, Gerald: „Christliche Philosophie im englischen Sprachraum“, in: Emerich Coreth SJ/Walter M. Neidl/Georg Pfligersdorffer (ed.): Christliche Philosophie im katholischen Denken des 19. und 20. Jahrhunderts III. Graz (et al.): Verlag Styria 1990, pp. $757-779$.

Ricken, Friedo: Religionsphilosophie. Stuttgart: Kohlhammer 2003.

Schneider, Hans Julius: „Wertstofftrennung?' Zu den sprachphilosophischen Voraussetzungen des Religionsverständnisses von Jürgen Habermas“, in: Rudolf Langthaler/ Hertha Nagl-Docekal (ed.): Glauben und Wissen. Wien: R. Oldenbourg 2007, pp. $155^{-185}$.

Taylor, Charles: Die Formen des Religiösen in der Gegenwart. Frankfurt: Suhrkamp ${ }^{4} 2013$. Thörner, Katja: William James' Konzept eines vernünftigen Glaubens auf der Basis religiöser Erfahrung. Stuttgart: Kohlhammer 2011.

Wittgenstein, Ludwig: Das Blaue Buch, in: Werkausgabe, Vol. 5, Frankfurt am Main: Suhrkamp 1984.

Wust, Peter: Ungewißheit und Wagnis. München: Kösel ${ }^{8} 1986$. 